\title{
Technology transfer and sustainability
}

\author{
A. A. Martins - Teresa M. Mata
}

Published online: 12 January 2010

(C) Springer-Verlag 2010

Once the main stakeholders agree on what sustainable development means and on what should be done to move towards it, new problems arise regarding how decisions will be implemented in practice. In other words, after defining the strategy and policies to best tackle these problems, questions appear concerning what are the best tactics, procedures or ways to move in the right direction or to meet some pre-defined goals. As a multidimensional area that touches all the aspects of the societal, environmental and economic spheres, with different time scales, many different aspects and conflicting interests have to be taken into account.

A classical example is the reduction of the environmental impact from the production and/or utilization of products or services in everyday life. On the consumer side, this can be done by changing their consumption patterns and habits, for example, by selective advertising, or by specific legislation or regulations that limit or even prohibit the use of certain products. However, these procedures have a limited impact and are strongly dependent on the consumer's response. On the production side, this can be accomplished by the implementation of cleaner production methodologies, for example with process integration, better logistical procedures, and development and implementation of new processes that use more environmentally friendly raw materials or milder conditions.

However, the development of new technologies and products, or improvement of existing ones, strongly depends on research made at private or public organizations. In many situations, there may be a significant gap

\footnotetext{
A. A. Martins $(\bowtie) \cdot$ T. M. Mata

Faculdade de Engenharia da Universidade do Porto (FEUP),

Rua Dr. Roberto Frias S/N, 4200465 Porto, Portugal

e-mail: amartins@fe.up.pt
}

between obtaining research results and their practical application. This is mainly due to the goals of the different partners involved in the process. Researchers are more interested in the creation of knowledge, industrialists are focused on its practical application and how that knowledge can help them be competitive, and consumers are more interested in improving their quality of life and reducing their environmental impact. The questions concerning the return on the investment made by companies in research and of intellectual property protection are also significant issues to consider.

The current emphasis on innovation and development of cleaner products and processes requires efficient forms of technology transfer and application of knowledge and technologies developed elsewhere in industrial and other practical settings. This way new or improved cleaner products and processes can be developed and implemented. This is not limited to companies and research institutions, but also extends to the transfer of knowledge and technology between developed and undeveloped countries. Good examples of this are the Technology Transfer Clearing House, and to a lesser extent the Clean Development Mechanism, defined under the United Nations Framework Convention for Climate Change.

The aforementioned questions were the main focus of a special topical symposium on "Best Practical Practices for Incorporation and Transfer of Sustainable Technologies in Industry" held in Porto, Portugal, on 7 May 2007, within the Annual Meeting of the NATO/SPS Pilot Study on "Clean Products and Processes."

This meeting involved not only delegates from this NATO/SPS Pilot Study, but also Portuguese researchers and people from industry. Practical examples were presented of successful transfer and application of knowledge and technologies developed within research institutions in 
practical contexts. Pertinent aspects and problems related to technology transfer were discussed between the participants, as well as an exchange of experiences in this area.

This issue of "Clean Technologies and Environmental Policy" contains some selected contributions to this special topical symposium. They include practical examples of technologies developed in university research and how they were applied in practice. For example, one considers a more efficient way of building bridges, another a lighter natural gas container, and another the application of optical technologies to practical problems. Other contributions deal with industrial problems involving the recycling of boron, an important raw material for some industrial processes with very limited natural resources, and the selection of metalcutting fluids. Combined together, the articles give an overview of the questions, problems and potential solutions involving the transfer of knowledge and/or technology to the development of cleaner products and processes. 